\title{
Asthma and respiratory allergy prevalence is still increasing among Finnish young adults
}

\author{
To the Editor:
}

Asthma and allergic diseases are a major public health problem, and their prevalence has been increasing in Finland and other developed countries since the 1960s [1,2]. Even though understanding of the origin of these conditions has improved in the last decades, tools for prevention are limited. The main aetiological hypotheses have varied over time. In the 1970-1980s, the main focus was on tobacco smoking and environmental pollution. In the 1990s, the "allergy epidemic" was linked with vaccinations, improved hygiene and reduced burden of infectious disease. During the last 10 years, reduced contact with environmental microbiota has been highlighted [3,4]. We report here the self-reported prevalence of asthma, hay fever or other allergic rhinitis and allergic eye symptom over a 15-year period among the Finnish adult population.

The National FINRISK Study has been carried out at 5-year intervals since 1972 to monitor the levels of noncommunicable disease risk factors in Finland [5]. Questions related to asthma and allergic symptoms were included in the study protocol first time in 1997. The present study population consisted of participants of the four last rounds of the study conducted in 1997, 2002, 2007 and 2012. In each survey year, a random sample of 10000 (8000 in 2012) 25-64-year-old men and women was taken. The participation rate varied from $65 \%$ to $72 \%$ and the total number of participants was 26939 . Data were collected using standardised self-administered questionnaires based on the European Community Respiratory Health Survey [6]. The study protocol was approved by the ethical committee and all participants gave informed consent.

Prevalence of asthma was assessed based on responses to the question of whether the respondent has asthma diagnosed by a physician. Hay fever or other allergic rhinitis and allergic eye symptoms were assessed by asking whether the respondent: 1) had never had the symptoms, 2) had had the symptoms during the past 12 months prior the survey, or 3) had had the symptoms but not during the past 12 months. In each study year, the data collection was performed between January and March, before the main pollen season.

Data were analysed adjusting for age and stratified by age groups. Statistical analyses were performed by the SAS 9.3 for Windows statistical package (SAS Institute, Cary, NC, USA). The significance of age-adjusted prevalence change over time was tested by the Chi-squared test and Wald confidence limits were used to calculate $95 \%$ confidence intervals. All analyses were done separately for men and women.

The age-adjusted prevalence of physician-diagnosed asthma increased from $6.1 \%$ to $9.5 \%$ in men ( $p$-value for the trend $<0.001)$ and from $7.8 \%$ to $10.8 \%$ in women $(\mathrm{p}<0.001)$ from 1997 to 2012 . The prevalence of hay fever/allergic rhinitis during the past 12 months prior the survey increased from $25.8 \%$ to $28.1 \%$ $(\mathrm{p}=0.084)$ in men and from $32.5 \%$ to $36.1 \%(\mathrm{p}=0.002)$ in women. The respective figures for allergic eye symptoms were $18.0 \%$ and $21.2 \%(p=0.019)$ for men and $25.9 \%$ and $28.3 \%(p<0.001)$ for women. Modest increase $(1-2 \%)$ was also observed in the numbers of those having had allergic nose or eye symptoms ever but not during the last 12 months prior the survey (data not shown). In the analysis stratified by age, the prevalence of asthma doubled, and allergic rhinitis and conjunctivitis increased markedly among those aged 25-44 years, but the prevalences remained at the same level among those aged 46-65 years (table 1).

The self-reported prevalence of asthma and allergy has been increasing in Finland during the past 15 years. The observed increase was mainly explained by the cohort effect, i.e. the increase affected mainly by the younger generations. A similar increase has been observed in many other industrialised countries, and the allergy and asthma gap between developed and developing world has become evident [2].

In connection with the 1997 study, we conducted a more detailed asthma and allergy substudy in the province of North Karelia in Finland and in its neighbouring area, the Pitkäranta region in the Republic of Karelia, Russia. Both self-reported asthma and allergic symptoms and objective markers of sensitisation were two to three times more common among the adults in North Karelia in Finland compared with Russian Karelia [7]. Re-examination of the same adult population 10 years later in 2007 revealed that the prevalence of symptoms and sensitisation had increased on the Finnish side but not on the Russian side [8]. The observed difference in the prevalence of allergic disorders between East and West Germany, Estonia and Sweden, and urban and rural Mongolia have provided somewhat comparable research opportunities and the results of these studies have been in line with the results of the Karelian Allergy Study [9-11]. 
TABLE 1 The prevalence of self-reported physician-diagnosed asthma and self-reported hay fever or other allergic rhinitis and allergic eye symptoms in the past 12 months prior to the survey among Finnish men and women aged $25-64$ years by age group

\begin{tabular}{ccccc} 
& \multicolumn{4}{c}{ Year } \\
\cline { 2 - 5 } & $\mathbf{1 9 9 7}$ & $\mathbf{2 0 0 2}$ & $\mathbf{2 0 0 7}$ & $\mathbf{2 0 1 2}$ \\
\hline Men aged 25-44 years & 1551 & 1773 & 1256 & 999 \\
Physician-diagnosed asthma & $5.2(4.1-6.3)$ & $6.4(5.2-7.5)$ & $7.6(6.1-9.0)$ & $10.5(8.6-12.4)$ \\
Hay fever/allergic rhinitis & $29.2(26.9-31.5)$ & $28.7(26.6-30.8)$ & $32.3(29.7-34.9)$ & $34.9(31.9-37.8)$ \\
Allergic eye symptoms & $20.2(18.2-22.2)$ & $22.8(20.9-24.8)$ & $24.0(21.6-26.4)$ & $27.9(25.1-30.7)$ \\
Men aged 45-64 years & 1844 & 2142 & 1591 & 1277 \\
Physician-diagnosed asthma & $6.9(5.7-8.0)$ & $7.3(6.2-8.4)$ & $6.4(5.2-7.6)$ & $8.7(7.1-10.2)$ \\
Hay fever/allergic rhinitis & $23.4(21.4-25.3)$ & $23.4(21.6-25.2)$ & $23.8(21.7-26.0)$ & $23.0(20.7-25.3)$ \\
Allergic eye symptoms & $16.6(14.9-18.3)$ & $17.1(15.5-18.7)$ & $17.7(15.8-19.6)$ & $16.1(14.1-18.1)$ \\
Women aged 25-44 years & 1804 & 2223 & 1557 & 1422 \\
Physician-diagnosed asthma & $5.4(4.4-6.5)$ & $8.0(6.9-9.1)$ & $10.3(8.8-11.8)$ & $10.4(8.7-12.1)$ \\
Hay fever/allergic rhinitis & $33.7(31.5-35.9)$ & $36.5(34.5-38.5)$ & $39.8(37.4-42.2)$ & $38.5(35.8-41.2)$ \\
Allergic eye symptoms & $26.1(24.1-28.2)$ & $29.7(27.8-31.6)$ & $33.8(31.4-36.1)$ & $29.6(27.1-32.2)$ \\
Women aged 45-64 years & 1959 & 2330 & 1789 & 1422 \\
Physician-diagnosed asthma & $9.9(8.6-11.3)$ & $10.3(6.3-11.6)$ & $9.3(7.9-10.6)$ & $11.2(9.5-12.8)$ \\
Hay fever/allergic rhinitis & $31.4(29.3-33.5)$ & $32.2(30.2-34.0)$ & $33.2(31.0-35.4)$ & $33.8(31.3-36.3)$ \\
Allergic eye symptoms & $25.8(23.8-27.7)$ & $24.6(22.9-26.4)$ & $27.1(24.9-29.2)$ & $27.0(24.7-29.4)$ \\
\hline
\end{tabular}

Data are presented as $\mathrm{n}$ or $\%(95 \% \mathrm{CI})$.

After the Second World War, Finland was a relatively poor country, more than half of population living in rural areas and one third working in agriculture and forestry. Urbanisation and lifestyle changes started to speed up in the 1960s. Rapid urbanisation; use of pasteurised milk, processed food and tap water; decreased contact with animals and soil; and sedentary lifestyle have taken place in a relatively short period of time. They may all have contributed to changes in human microbiomes and development of immune tolerance and protection against inappropriate inflammatory responses, including allergy $[12,13]$.

Our data are based on self-reports only. However, the data were collected repeatedly and systematically using a standardised and internationally validated set of questions [6]. The participation rate in the surveys was relatively high. In the first 1997 survey, we also had an opportunity to compare the questionnaire data with biological markers of sensitisation in a subsample [7]. We do not have comparable data on the prevalence of chronic obstructive pulmonary disease (COPD) because COPD was included in the questionnaire only in the last survey round. However, in 2012, self-reported COPD was rare: the prevalence was practically zero in the younger age group and about $1 \%$ in the older age group. The low figures probably indicate underdiagnosis and poor awareness of the disease but also a lack of any diagnostic shift from asthma to COPD. The prevalence of smoking decreased among men and women in the younger age group and among men in the older age group but remained at the same level among the older women over the 15-year period, being $28 \%, 20 \%, 24 \%$ and $18 \%$ in 2012 , respectively.

New allergic sensitisations are uncommon after young adulthood and it is necessary to target the preventive measures to the young generation. One of the main goals of the Finnish allergy programme 2008-2018 is to stop the increase in allergy prevalence [14]. The trend up to 2012 has been upwards but if the ongoing action plan works, the trend may slow down till the next survey in 2017. Furthermore, rapidly urbanising countries, such as India and China, are now facing the allergy epidemic that started to become evident in the industrialised European countries some 40 years ago. Therefore, better understanding of the aetiology of allergy and asthma and development of effective preventive methods are essential for global health.

0 $@$ @ERpublications

Asthma and respiratory allergy are still increasing in Finland, which is mainly explained by the cohort effect http://ow.ly/UwhND

Pekka Jousilahti ${ }^{1}$, Tari Haahtela ${ }^{2}$, Tiina Laatikainen ${ }^{1,3}$, Mika Mäkelä ${ }^{2}$ and Erkki Vartiainen ${ }^{1}$

${ }^{1}$ National Institute for Health and Welfare, Dept of Health, Helsinki, Finland. ${ }^{2}$ Helsinki University Central Hospital, Skin and Allergy Hospital, Helsinki, Finland. ${ }^{3}$ University of Eastern Finland, Institute of Public Health and Clinical Nutrition, Kuopio, Finland.

Correspondence: Pekka Jousilahti, National Institute for Health and Welfare, Dept of Health, PO Box 30, 00271 Helsinki, Finland. E-mail: pekka.jousilahti@thl.fi 
Conflict of interest: None declared.

\section{References}

1 Haahtela T, Lindholm H, Björksten F, et al. The prevalence of asthma in Finnish young men. BMJ 1990; 301: 266-268.

2 Asher MI, Montefort S, Björkstén B, et al. Worldwide time trends in the prevalence of symptoms of asthma, allergic rhinoconjunctivitis, and eczema in childhood: ISAAC Phases One and Three repeat multicountry cross-sectional surveys. Lancet 2006; 368: 733-743.

3 Ege MJ, Mayer M, Normand AC, et al. Exposure to environmental microorganisms and childhood asthma. $N$ Engl J Med 2011; 364: 701-709.

4 Haahtela T, Laatikainen T, Alenius $\mathrm{H}$, et al. Hunt for the origin of allergy - comparing the Finnish and Russian Karelia. Clin Exp Allergy 2015; 45: 891-901.

5 Borodulin K, Vartiainen E, Peltonen M, et al. Forty-year trends in cardiovascular risk factors in Finland. Eur J Public Health 2015; 25: 539-546.

6 Burney PG, Luczynska C, Chinn S, et al. The European Community Respiratory Health Survey. Eur Respir J 1994; 7: 954-960.

7 Vartiainen E, Petäys T, Haahtela T, et al. Allergic diseases, skin prick test responses, and IgG levels in North Karelia, Finland, and the Republic of Karelia, Russia. J Allergy Clin Immunol 2002; 109: 643-648.

8 Laatikainen T, von Hertzen L, Koskinen JP, et al. Allergy gap between Finnish and Russian Karelia on increase. Allergy 2011; 66: 886-892.

9 von Mutius E, Fritzsch C, Weiland SK, et al. Prevalence of asthma and allergic disorders among children in united Germany: a descriptive comparison. BMJ 1992; 305: 1395-1399.

10 Riikjärv MA, Julge K, Vasar M, et al. The prevalence of atopic sensitization and respiratory symptoms among Estonian schoolchildren. Clin Exp Allergy 1995; 25: 1198-1204.

11 Viinanen A, Munhbayarlah S, Zevgee T, et al. Prevalence of asthma, allergic rhinoconjunctivitis and allergic sensitization in Mongolia. Allergy 2005; 60: 1370-1377.

12 Haahtela T, Holgate ST, Pawankar R, et al. The biodiversity hypothesis and allergic disease: world allergy organization position statement. World Allergy Organ J 2013; 6: 3.

13 Hesla HM, Stenius F, Jäderlund L, et al. Impact of lifestyle on the gut microbiota of healthy infants and their mothers - the ALADDIN birth cohort. FEMS Microbiol Ecol 2014; 90: 791-801.

14 Haahtela T, von Hertzen L, Mäkelä M, et al. Finnish Allergy Programme 2008-2018 - time to act and change the course. Allergy 2008; 63: 634-645.

\section{Obstructive sleep apnoea as a risk factor for osteopenia and osteoporosis in the male population}

To the Editor:

Obstructive sleep apnoea (OSA) is a sleep disorder characterised by recurrent apnoea events leading to hypoxia, hypercapnia and sleep disruption [1]. OSA represents a growing health problem mainly affecting men; in fact, its prevalence in the adult male population is between $4 \%$ and $24 \%$ [1-3].

It was recently demonstrated that OSA may lead to a deficient vitamin D status inducing a secondary hyperparathyroidism, which may produce the demineralisation of the skeleton and a reduction of the bone mineral density (BMD) [3]. In keeping with this observation, recent studies documented reduced BMD in OSA patients, thus speculating that OSA may represent a risk factor for bone resorption [4-6]. However, the evidence proposed so far is controversial due to differences in patient populations, study designs and definition of OSA; principally, since physical activity and body mass index (BMI) can negatively influence $\mathrm{BMD}$, major limitations of the previous studies are the lack of physical activity assessment and absence of BMI-matched controls $[7,8]$.

Therefore, the aim of the present study was to evaluate bone homeostasis in a large cohort of male OSA patients compared to male controls matched for age, BMI and physical activity, also investigating and correlating the measured BMD to polygraphic parameters, Epworth Sleepiness Scale (ESS) scores and serum biomarker levels, such as vitamin $\mathrm{D}$, parathormone $(\mathrm{PTH})$, calcium, fibrinogen and C-reactive protein (CRP). 